\title{
The Total Release Method for FIB In-situ TEM Sample Preparation
}

\author{
L. Zaykova-Feldman and T.M. Moore
}

Omniprobe, Inc., 10410 Miller Road, Dallas, TX 75238

The use of focused ion beam (FIB) systems has become the method of choice for sitespecific TEM sample preparation for integrated circuit process control and failure analysis. This requires the fabrication of an electron-transparent $(<50 \mathrm{~nm}$ thickness) region of interest on a sample that has been excised from the wafer or die by ion beam milling. The use of the FIB offers advantages over conventional mechanical TEM sample preparation. The dual-beam FIB offers the ability to inspect the sample with SEM resolution while using the ion beam to thin the sample to the thickness required for TEM inspection without sacrificing the wafer. This is especially attractive for $300 \mathrm{~mm}$ processing. This method for performing the entire TEM sample preparation within the FIB is known as "in-situ lift-out" and is based on use of a chamber-mounted nanomanipulator and FIB-induced material deposition [1-5].

This article describes the "Total Release" method for in-situ lift-out which is designed to maximize throughput in a process control application. In this method, the ion beam is used to make a substantially U-shaped first cut into the wafer, at least partially surrounding the target. Next, the wafer is re-positioned with respect to the ion beam, and the ion beam is positioned at an angle in the range of 45 to 60 degrees to the plane of the wafer. A second cut is made in the wafer with the ion beam, undercutting the target, so that a sample is completely released from the wafer. Then the probe is fixed to the released sample, preferably with ion-beam deposition, so the sample and the wafer can be separated. The order of cuts can be reversed, so the ion beam is positioned at an angle less than 90 degrees to the plane of the wafer and the ion beam is used to make a first cut in the wafer, undercutting the target. Next, the wafer is repositioned with respect to the ion beam by turning the wafer approximately 180 degrees, and the ion beam is repositioned to substantially normal incidence to the plane of the wafer. The ion beam then makes a U-shaped second cut into the wafer, at least partially surrounding the target and intersecting the first cut, so that the sample is completely released from the wafer. A probe is attached to the sample as before. The sequence of steps is shown in Figure 1.

The Total Release method has several advantages for high-throughput TEM sample preparation. It allows for instant and definitive indication that the sample release procedure has been completed successfully, thus saving time. If pressure is applied by the probe to the sample during the final milling procedure, the mechanical displacement of the tip of the probe, or a change in mechanical load measured by a sensitive strain gauge inside the probe shaft of the nanomanipulator, can be used to indicate that the sample release procedure has been successfully completed [6]. This same strain gauge can be used to detect contact between the tip of the probe and the sample in an automated process. Such a detection method is independent of electrical continuity effects and 
functions efficiently on any type of sample surface. Alternatively, the probe tip need not apply pressure to the sample during the final milling procedure, but can be positioned directly over the sample to prevent the sample from leaving the pit from which it was released due to (for example) static electric interactions. In this approach any movement of the sample within the pit can be used as an indicator that the sample release procedure has been successfully completed.

Since the probe tip is not attached to the sample prior to the final cut, a premature lift-out attempt can be avoided, and re-deposition in the initial stage of the lift-out process can be more easily cleared without having to detach the probe tip. A probe tip-based approach is more suitable for high-throughput because of the reduced amount of ion milling and because there is less risk of loosing or damaging the unique sample on its way to the TEM [6].

[1] L.A. Giannuzzi et al., "FIB Lift-Out Specimen Preparation Techniques", in Introduction to Focused Ion Beams: Instrumentation, Theory, Techniques, and Practice, L.A. Giannuzzi, F.A. Stevie (eds), Springer-Verlag New York, 201 (2004).

[2] T. Kamino et al., "A FIB Microsampling Technique and a Site Specific TEM Specimen Preparation Method", in Introduction to Focused Ion Beams: Instrumentation, Theory, Techniques, and Practice, L.A. Giannuzzi, F.A. Stevie (eds), Springer-Verlag New York, 229 (2004).

[3] T. Ohnishi et al., U.S. Patent 5270552.

[4] T.M. Moore et al., U.S. Patent 6420722.

[5] T.M. Moore, U.S. Patent 6570170.

[6] Patent pending.

[7] The authors wish to thank to R. Kruger and C.D. Hartfield for their dedicated efforts.
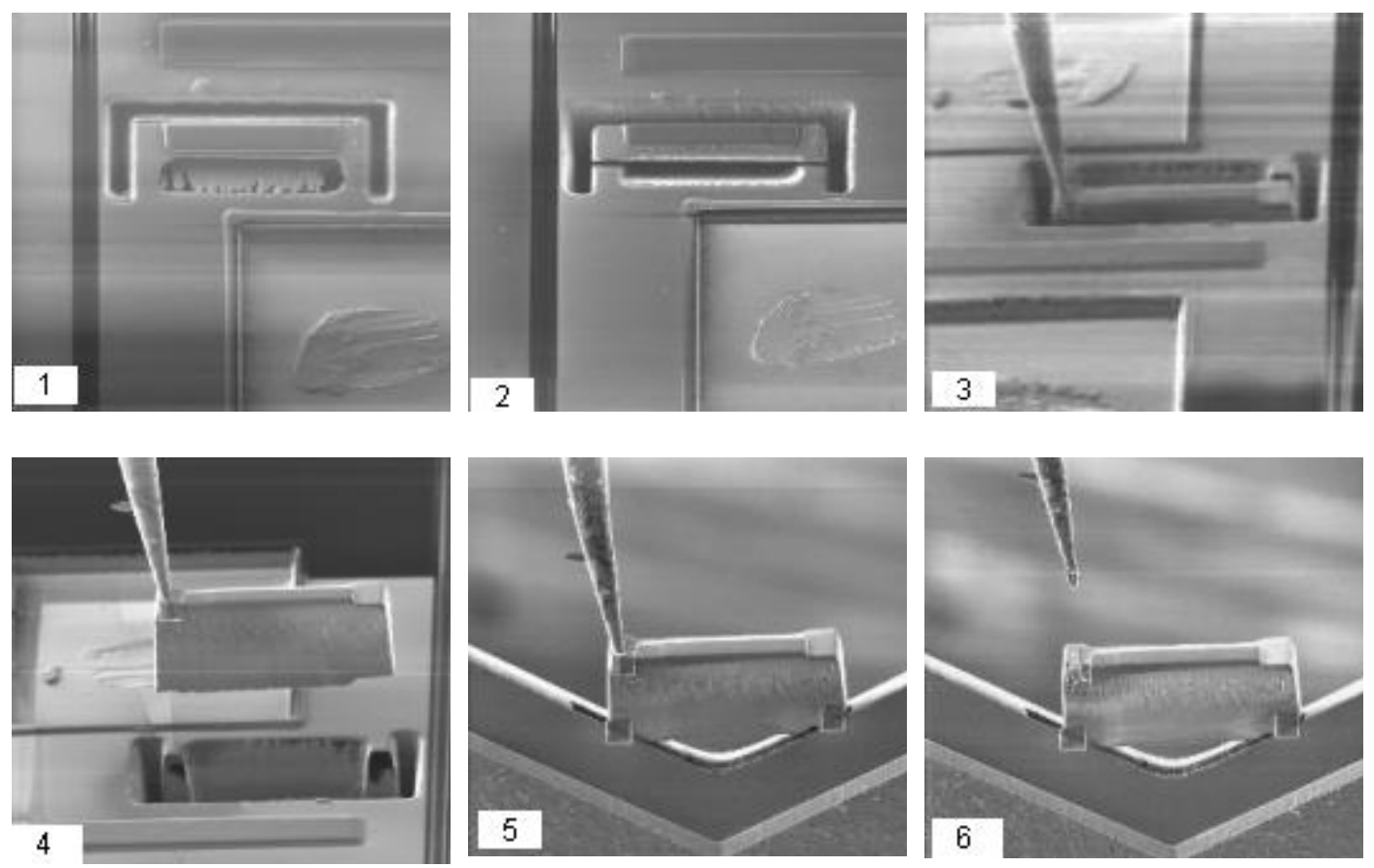

Fig.1. Steps of In-situ Lift-out Total Release method. 